\section{PSQ-135 SAFETY RELATED TO PSYCHOGERIATRIC PATIENTS. ONE-YEAR PROSPECTIVE STUDY}

${ }^{1} \mathrm{M}$ Hernandez*${ }^{*}{ }^{2} \mathrm{C}$ Mestres, ${ }^{3} \mathrm{~J}$ Junyent, ${ }^{4} \mathrm{P}$ Modamio, ${ }^{5} \mathrm{C}$ Fernandez, ${ }^{5} \mathrm{EL}$ Mariño ${ }^{1}$ Blanquerna Health Science_ Ramon Llull University, Pharmacy, Barcelona, Spain; ${ }^{2}$ School of Health Sciences Blanquerna. University Ramon Llull, Health Sciences, Barcelona, Spain; ${ }^{3}$ Grup Mutuam, Hospital Güell, Barcelona, Spain; ${ }^{4}$ Department of Pharmacy and Pharmaceutical Technology- and Physical Chemistry- Faculty of Pharmacy and Food Sciences- University of Barcelona- Barcelona- Spain, Clinical Pharmacy and Pharmacotherapy Unit, Barcelona, Spain; ${ }^{5}$ Department of Pharmacy and Pharmaceutical Technology- and Physical Chemistry- Faculty of Pharmacy and Food Sciences- University of Barcelona, Clinical Pharmacy and Pharmacotherapy Unit, Barcelona, Spain

10.1136/ejhpharm-2019-eahpconf.568

Background Patient safety is the most important concern for healthcare professionals, patients and healthcare systems. Adverse drug events (ADEs) are a common cause of hospitalisation and occur with increasing frequency in hospital as patients age.

Purpose Determine the ADEs at admission and during the stay in a psychogeriatric unit.

Material and methods One-year prospective cross-sectional study (July 2015-2016), in a psychogeriatric ward (21 beds). Included patients with dementia, admitted presenting neuropsychiatric/behavioural and psychological symptoms in dementia (BPSD). Excluded patients with previous psychiatric illness or palliative conditions.

Prescription information: Aegerus and Catalonia clinical record HC3. Variables: demographics, diagnoses, Global Deterioration Scale (GDS-R), functional assessment (Barthel Index), fall risk (Downton $\mathrm{JH}$ and Tinetti). Anticholinergic risk level (Drug Burden Index (DBI). ADE assessment: causality by Naranjo algorithm, severity by classification system of the Institute for Healthcare Improvement and preventability by the Schumock-Thornton algorithm. Drugs classification by the Anatomical Therapeutic Chemical Code (ATC).

Results Sixty patients (60\% females), age: 84.8 years (68-96). Dementia: unidentified (43\%), Alzheimer's (31\%), Lewy bodies (8\%), vascular (8\%), mixed (5\%) and others (6\%). GDS-R $4.5( \pm 1.2)$, moderate cognitive impairment. Barthel Index $43.8( \pm 23.9)$, moderate dependence. Patients comorbidities $4.8( \pm 1.6)$. Drugs/patient $9.03( \pm 3.12)$. DBI high risk in $69 \%$ of the patients. High risk of falls, Tinetti $(15.5 \pm 8)$ and Downton test $(4.5 \pm 1.3)$.

Sixty-eight ADEs (53 patients, 81.5\%, 22.6\% more than one). $73.5 \%$ not related to falls. From this $80 \%$ were related to the ATC nervous system (46\% (23 ADE) psycholeptics). Naranjo algorithm one definite (2\%), probable 45 (90\%) and possible four (8\%). Severity Category E: temporary harm to the patient and required intervention in 34 (68\%) and F: temporary harm to the patient and required initial or prolonged hospitalisation in 16 (32\%). Schumork-Thornton test $58 \%$ (29) of the ADE were preventable, possibly preventable $6 \%$ (three) and unavoidable 36\% (18). Main ADE drowsiness/somnolence $27.7 \%, 12.8 \%$ weakness and hypoactivity and $10.7 \%$ hypotension. DBI significant differences related to fall as ADE result (fall group 1.288 \pm 0.79 , non-fall group $0.95 \pm 0.71$ $\mathrm{p}=0.05)$.

Conclusion The balance between effective treatments and safety is complex in these patients. Mostly ADE are related to the pharmacological treatment of this BPSD.

Anticholinergic load is a determinant for a specific ADE and were related to falls, the worst consequence in this patient, clinical and economic impact.
REFERENCES AND/OR ACKNOWLEDGEMENTS

No conflict of interest.

\section{PSQ-136 A PHARMACEUTICAL CARE PROGRAMME IN PATIENTS UNDERGOING CARDIAC SURGERY}

S Ibañez Garcia*, X Garcia Gonzalez, A Ribed Sanchez, A de Lorenzo-Pinto, A Giménez Manzorro, C Ortega-Navarro, B Marzal Alfaro, E Garcia Martin, A Herranz Alonso, M Sanjurjo Sáez. Hospital General Universitario Gregorio Marañon, Pharmacy, Madrid, Spain

\subsection{6/ejhpharm-2019-eahpconf.569}

Background The preoperative setting is an area with high risk for medication errors with potentially severe consequences. Strategies to reduce the incidence of medication errors are needed to guarantee the safety of these patients.

Purpose To describe the implementation of a pharmaceutical care programme (PCP) in patients undergoing cardiac surgery and its results in preventing medication errors.

Material and methods The comprehensive PCP was implemented in July 2018. Telephonic pharmacists' clinical interviews took place the week prior to cardiac surgery. Patients' complete medication list including over the counter and herbal products was compiled during the interview and medical instructions for adequate preoperative drug management were reinforced. Patients were admitted to the hospital at least 36 hours before the surgery, where medication management was supervised by ward personnel. At the time of admission, the ward pharmacist performed the medication reconciliation and suggested any further adjustments deemed necessary.

Observational prospective study. Time of study: July 2018 to September 2018. Pharmacists' interventions classified according to Overhage classification and the severity of medication errors accordingto NCC MERP were analysed.

Results Fifty-one patients were included, mean age 67.7 years, $60.8 \%$ males. Patients received an average of 7.1 (SD 3.1) chronic drugs. Forty patients (78.4\%) were taking drugs that needed to be discontinued before surgery. Nineteen patients $(37.3 \%)$ were on anticoagulants and 19 were taking antiplatelet drugs. Pre-operative drug management was correct in all cases.

Thirty-eight pharmacist interventions were made at the time of admission, classified as: wrong dose (47.4\%), drug omission (31.6\%), wrong frequency of administration (10.5\%), wrong drug $(7.9 \%)$ and wrong dosage form $(2.6 \%)$. Overall acceptance of pharmacy interventions was $89.5 \%$.

According to the severity of medication errors, 30 (78.9\%) errors were serious $(\mathrm{E} / \mathrm{F})$, and eight $(21.1 \%)$ classified as error without harm $(\mathrm{C} / \mathrm{D})$.

Regarding health outcomes, no surgeries had to be postponed due to wrong perioperative medication management. The mean length of hospital stay was 16 days (2-60). The readmission rate at 30 days was $3.9 \%(n=2)$.

Conclusion The PCP in patients undergoing cardiac surgery was successfully implemented, ensuring a correct preoperative drug management. We intercepted 0.8 medication errors per patient included in the programme. The errors were mostly serious.

\section{REFERENCES AND/OR ACKNOWLEDGEMENTS}

No conflict of interest. 